\title{
Oscillations of Respiration, Adenine Nucleotide Levels and Heat Evolution in Synchronous Cultures of Tetrahymena pyriformis ST Prepared by Continuous-flow Selection
}

\author{
By DAVID LLOYD, CAROL A. PHILLIPS* AND MICHAEL STATHAM \\ Department of Microbiology, University College, Newport Road, Cardiff CF2 1TA
}

(Received 2 December 1977)

\begin{abstract}
Respiration rates, adenine nucleotide levels and heat production were measured during exponential asynchronous growth of Tetrahymena pyriformis and in synchronous cultures prepared by continuous-flow selection. In synchronous cultures rates of oxygen uptake rose to maxima three times in each cell cycle. The ATP pool oscillated in phase with respiratory activity. The ADP and AMP pools oscillated in phase with each other, but out of phase with ATP, and the rate of heat production also showed fluctuations. Values calculated for adenylate energy charge were low, increasing from less than 0.2 to more than 0.4 during the mid-exponential phase of growth in an asynchronous culture, and oscillating over a range from 0.34 to 0.47 in a synchronous culture.
\end{abstract}

\section{INTRODUCTION}

Respiratory rates have been measured through the cell cycles of many eukaryotic micro-organisms (see Lloyd, 1974), but satisfactory explanations have not often been presented for the complex patterns that have been reported. Parallel measurements of other parameters of energy metabolism are necessary for interpretation of the observed respiratory patterns.

Adenylate energy charge serves as an indicator of the state of energy metabolism (Atkinson \& Walton, 1967). In many prokaryotic micro-organisms the energy charge is strongly stabilized during growth (Dietzler et al., 1974; Swedes, Sedo \& Atkinson, 1975; Niven, Collins \& Knowles, 1977). On the other hand, wide variations in values for adenylate energy charge have been observed in some eukaryotic systems at different stages during the cell cycle (Edwards, Statham \& Lloyd, 1975), during the growth of batch cultures (Edwards \& Lloyd, 1977) and on carbon starvation (Ball \& Atkinson, 1975; Lloyd et al., 1978).

Measurements of nucleoside phosphates carried out on heat-shock synchronized Tetrahymena pyriformis suggest that there are cyclic variations in nucleotide concentration after heat treatment (Stocco \& Zimmerman, 1975; De Balros, De Castro \& De Castro, 1973). However, since nucleoside triphosphates are extremely sensitive to heat treatment (Scherbaum et al., 1962), measurements on synchronous cultures produced by a selection method (Lloyd et al., 1975; Phillips \& Lloyd, 1978) should help to elucidate the patterns of synthesis in a relatively undisturbed cell cycle. In the present investigation, changes in adenylate nucleotides have been studied alongside changes in respiration and heat production.

\footnotetext{
* Present address: Department of Radiopharmacology, Institute of Cancer Research, Royal Cancer Hospital, Sutton, Surrey SM2 5PX.
} 


\section{METHODS}

Maintenance and growth of the organism. Tetrahymena pyriformis strain ST (obtained from Dr Y. Suyama, Department of Biology, University of Pennsylvania, Philadelphia, U.S.A.) was maintained and grown as described previously (Lloyd et al., 1971).

Preparation of synchronous cultures. Synchronous cultures were prepared by continuous-flow size selection (Lloyd et al., 1975). The rotor speeds and flow rates used to obtain 10\% of the original population in the effluent are given in the figure legends.

Assessment of synchrony. The degree of synchrony was assessed by the synchrony index $(F)$ of Blumenthal \& Zahler (1962), calculated from the equation:

$$
F=\left(N / N_{0}\right)-2^{t / g}
$$

in which $F$ has a maximum value of 1.0 in a culture exhibiting perfect synchrony, $N$ is the number of organisms at time $t, N_{0}$ is the number of organisms at zero time, and $g$ is the mean generation time. In the figures, $F_{1}$ and $F_{2}$ represent the indices of the first and second doublings respectively.

Analytical methods. Oxygen uptake rates were measured polarographically using a Beckman oxygen electrode in a closed reaction vessel $(1.1 \mathrm{ml}$ total volume). All measurements were made with cells in culture medium at $29^{\circ} \mathrm{C}$.

Heat evolution was measured using a heat conduction flow microcalorimeter (LKB 10700-1) as described by Poole, Lloyd \& Kemp (1973), except that the flow rate for the culture medium and air into the reaction vessel was $54 \mathrm{ml} \mathrm{h}^{-1}$.

Measurement of adenine nucleotides was as described by Edwards et al. (1975) using luciferase assays on extracts prepared from $1.0 \mathrm{ml}$ culture samples by chloroform extraction. Samples were rapidly and directly released into tubes containing chloroform, and rapid mixing using a 'Whirlimixer' ensured that quenching occurred in about $0.2 \mathrm{~s}$. Identical results were obtained using the butanol method of extraction (Edwards et al., 1975). ATP, ADP and AMP were estimated by the method of Chapman, Fall \& Atkinson (1971) using ATP as a calibration standard. Values for adenylate charge were calculated according to the definition of Atkinson \& Walton (1967):

$$
\text { Adenylate charge }=\frac{\mathrm{ATP}+\frac{1}{2} \mathrm{ADP}}{\mathrm{ATP}+\mathrm{ADP}+\mathrm{AMP}}
$$

The lower limit of sensitivity of the ATP assay was $0.1 \mathrm{pmol}$ in a total volume of $0.21 \mathrm{ml}$. Coefficients of variation $(V)$ calculated from five determinations of adenine nucleotides were ATP $5 \%$, ADP $6 \%$, AMP $7 \%$, and the corresponding coefficient for adenylate charge was $3 \%$, where $V=($ standard deviation $/$ mean $) \times$ $100 \%$.

\section{RESULTS}

\section{Changes in respiration and adenine rucleotide pools during asynchronous growth}

Figure 1 shows the changes in oxygen uptake rates and adenine nucleotide pools during mid-exponential phase growth of an asynchronous culture. The ATP pool (Fig. $1 b$ ) increased continuously, ADP concentration remained at about $0 \cdot 2 \mathrm{nmol}$ per ml culture, while AMP and total adenylates decreased. Thus values for adenylate energy charge (Fig. $1 c$ ) were low, but increased from less than 0.2 to greater than 0.4 during the experiment. The respiration rate (Fig. $1 d$ ) increased continuously, doubling every $2 \cdot 5 \mathrm{~h}$.

\section{Changes in respiration and adenine nucleotide pools in synchronous cultures}

Figure 2 shows the patterns of increases in respiration rate, adenine nucleotides and adenylate energy charge during the growth of a synchronous culture; the results were similar in five separate experiments. Maxima in respiration rates (Fig. $2 b$ ) occurred at (mean values \pm standard deviations) $0.42 \pm 0.05,0.65 \pm 0.04$ and $0.89 \pm 0.03$ of the cell cycle in the first cycle after selection, and at $0 \cdot 27 \pm 0.04,0 \cdot 57 \pm 0.08$ and $0.80 \pm 0.05$ in the second cycle (seven experiments; cell cycle time $=0$ to $1 \cdot 0 ; 0=$ mid-point of cell division). No such respiratory oscillations were observed in exponentially growing cultures (Fig. 1) which suggests that they are cell cycle-related. Oscillations in the pool sizes of ATP (Fig. $2 c$ ), ADP (Fig. $2 d$ ) and AMP (Fig. 2e) accompanied the oscillations in respiratory rates (Fig. 2b) 


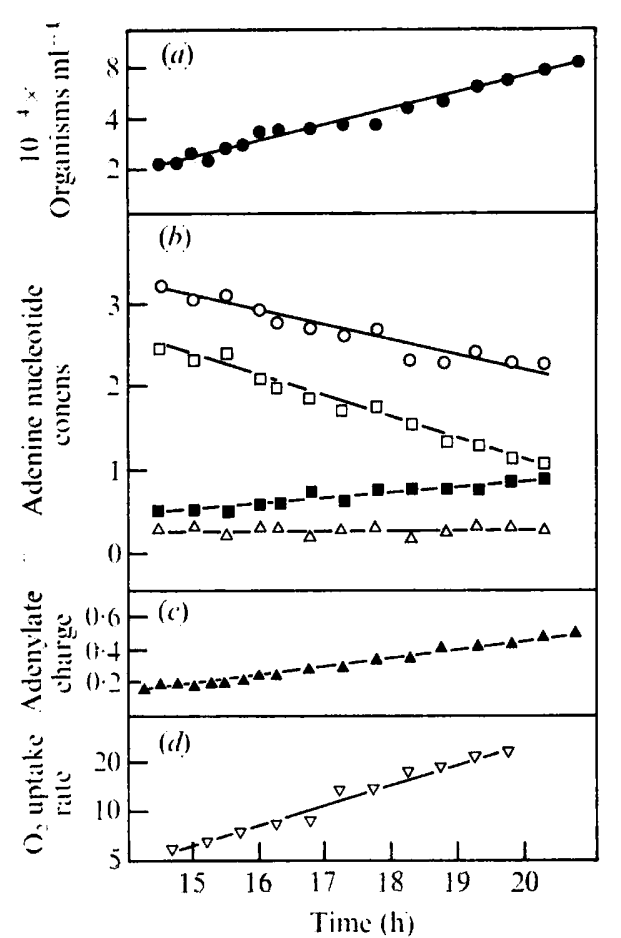

Fig. 1

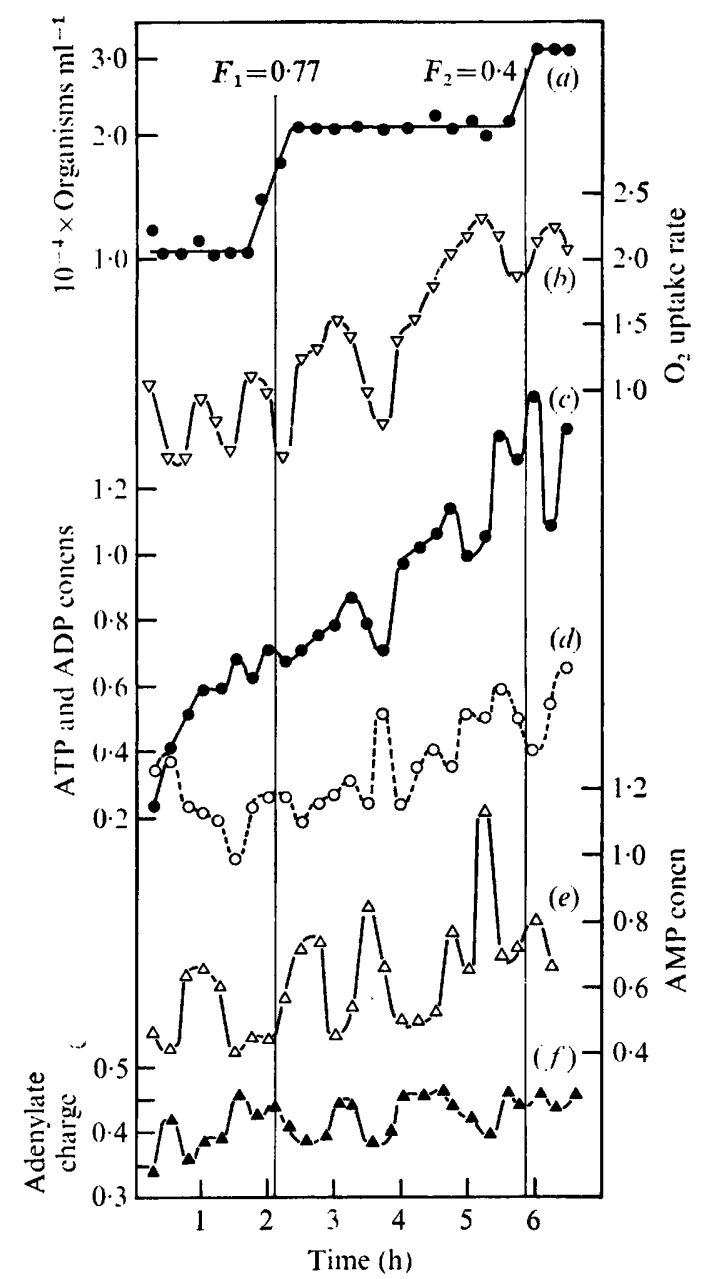

Fig. 2

Fig. 1. Changes in oxygen uptake rates and adenine nucleotide levels during exponential growth of $T$. pyriformis. Samples were removed at frequent intervals from a mid-exponential phase batch culture. Oxygen uptake was measured in an oxygen electrode, and $1 \mathrm{ml}$ portions were rapidly mixed with $0.3 \mathrm{ml}$ chloroform for adenine nucleotide estimations. (a) Cell numbers. (b) $\bigcirc$, Total adenylates; $\square$, AMP; $\square$, ATP; $\triangle$, ADP; all expressed as nmol (ml culture) $)^{-1}$. (c) Adenylate charge. $(d)$ Oxygen uptake rates, expressed as $\mathrm{nmol} \mathrm{O}_{2} \min ^{-1}(\mathrm{ml} \text { culture })^{-1}$. Inoculation was at time 0 .

Fig. 2. Changes in oxygen uptake rates and adenine nucleotide levels during growth of a synchronous culture of $T$. pyriformis. Samples were removed at frequent intervals for oxygen uptake measurements and $1 \mathrm{ml}$ portions were mixed with $0.3 \mathrm{ml}$ chloroform for adenine nucleotide estimations. Percentage selection from the exponentially growing culture used for the preparation of the synchronous culture by continuous-flow size selection was $10 \%$ : inlet flow rate, $1.5 \mathrm{I} \mathrm{min}^{-1}$; rotor speed, $1750 \mathrm{rev} . \mathrm{min}^{-1}$. (a) Cell numbers; (b) oxygen uptake rates, expressed as nmol $\mathrm{O}_{2} \mathrm{~min}^{-1}(\mathrm{ml}$ culture $)^{-1} ;(c)$ ATP; $(d)$ ADP; $(e)$ AMP; $(f)$ adenylate charge. All adenine nucleotides are expressed as nmol (ml culture) ${ }^{-1}$.

and led to marked fluctuations in the adenylate charge values (Fig. $2 f$ ). Total adenylates (not shown) also fluctuated about an exponential overall increase. The peak-trough amplitudes of the observed oscillations (expressed as a percentage of the pool sizes at the minima) were as much as $40 \%, 50 \%$ and $70 \%$ for ATP, ADP and AMP respectively; the range of adenylate energy charge values was 0.34 to 0.47 . The periodicities were similar in cultures exhibiting different synchrony indices, but the amplitude of the oscillations was 


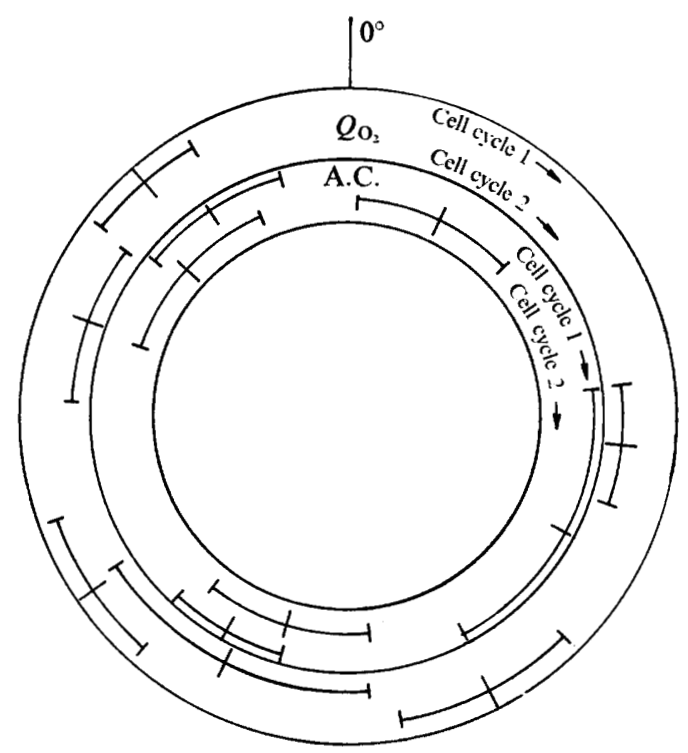

Fig. 3. Cell cycle map of the timings of maxima of oxygen uptake rates $\left(Q_{0_{2}}\right)$ and adenylate charge (A.C.) in T. pyriformis. The phase angles of the oscillatory components are plotted with respect to the mid-points of cell division (at a phase angle of $0^{\circ}$ ). For each component, the outer circle indicates maxima in the first cell cycle, and the inner circle, those in the second cell cycle. The results are expressed as mean values \pm standard deviations (seven experiments for respiration rates, five for adenylate charge).

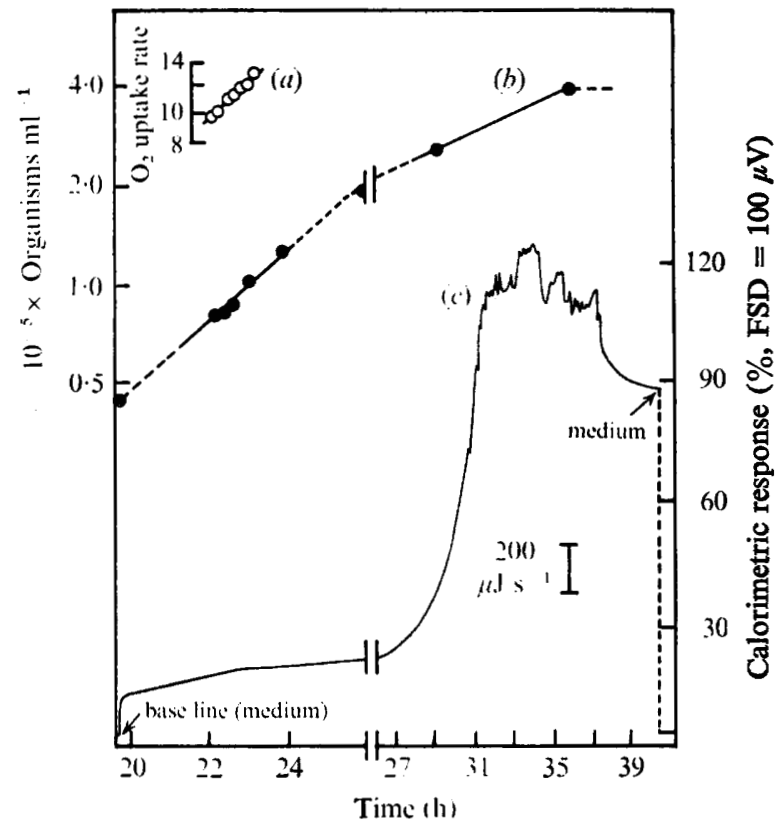

Fig. 4. Oxygen uptake and heat evolution of an asynchronous culture of $T$. pyriformis. Measurements of oxygen uptake $\left[a\right.$; expressed as $\left.\mathrm{nmol} \mathrm{O}_{2} \min ^{-1}(\mathrm{ml} \text { culture })^{-1}\right]$ were made on culture samples removed at intervals during the phase of rapid growth. Heat production $(c)$ was measured continuously to the onset of the stationary phase of growth using a flow microcalorimeter. Inoculation was at time 0 . 


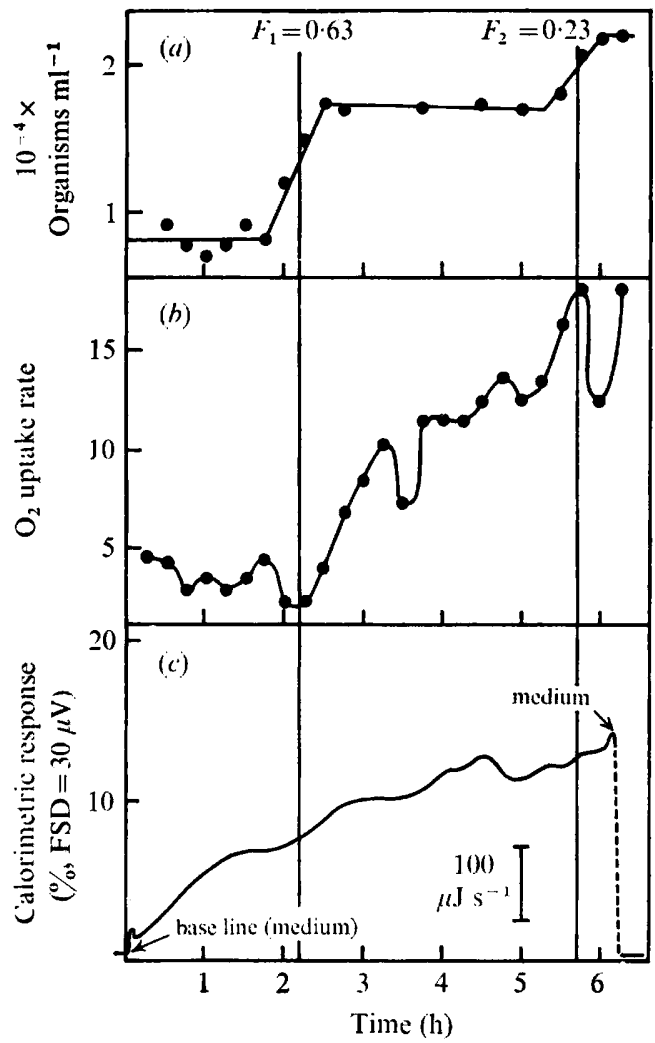

Fig. 5. Oxygen uptake and heat evolution of a synchronous culture of T. pyriformis. Measurements of oxygen uptake $\left[b\right.$; expressed as $\left.\mathrm{nmol} \mathrm{O}_{2} \min ^{-1}(\mathrm{ml} \text { culture })^{-1}\right]$ were made on culture samples removed at frequent intervals from a synchronous culture. Heat evolution $(c)$ was measured continuously using a flow microcalorimeter. Percentage retention from the exponentially growing culture used for the preparation of the synchronous culture by continuous-flow size selection was $10 \%$ : inlet flow rate, $1.51 \mathrm{~min}^{-1}$, rotor speed, $2000 \mathrm{rev} . \mathrm{min}^{-1}$.

attenuated in cultures with a low degree of synchrony. Calculation of ATP/ADP ratios for the experiment shown gave values varying between $0 \cdot 7$ and $8 \cdot 4$. The cell cycle timings for maxima of adenylate energy charge values were (mean values \pm standard deviations) $0.33 \pm 0.09,0.58 \pm 0.04$ and $0.91 \pm 0.05$ in the first cell cycle after selection, and $0.07 \pm 0.06$, $0.54 \pm 0.06$ and $0.86 \pm 0.06$ in the second cell cycle (five experiments; cell cycle time $=0$ to $1 \cdot 0 ; 0=$ mid-point of cell division). These timings are shown on a circular cell cycle map in Fig. 3, together with those for maxima of respiration rates.

\section{Heat evolution in asynchronous and synchronous cultures}

The rate of heat production increased continuously through the exponential phase of growth in an asynchronous culture (Fig. 4). The rate of increase declined when the population reached $0.9 \times 10^{5}$ organisms $\mathrm{ml}^{-1}$ and then remained low until the count reached $2 \times 10^{5}$. At this population a transition to a slower rate of growth occurred (Phillips \& Lloyd, 1978; Fig. $4 b$ ) and rates of heat evolution increased rapidly. As the culture approached the stationary phase of growth, heat evolution levelled off and exhibited chaos before entering a phase of decline. In the rapid (mid-exponential) phase of growth the respiration rate increased with cell density (Fig. $4 a$ ); at a population density of $8 \cdot 1 \times 10^{4}$ organisms $\mathrm{ml}^{-1}$, the heat production was $1620 \mu \mathrm{J}$ per nmol oxygen consumed and the rate of heat production per $10^{4}$ organisms was $33 \cdot 3 \mu \mathrm{J} \mathrm{s}{ }^{-1}$.

In contrast, the time course of the rate of heat evolution in a synchronous culture (Fig. $5 c$ ) 


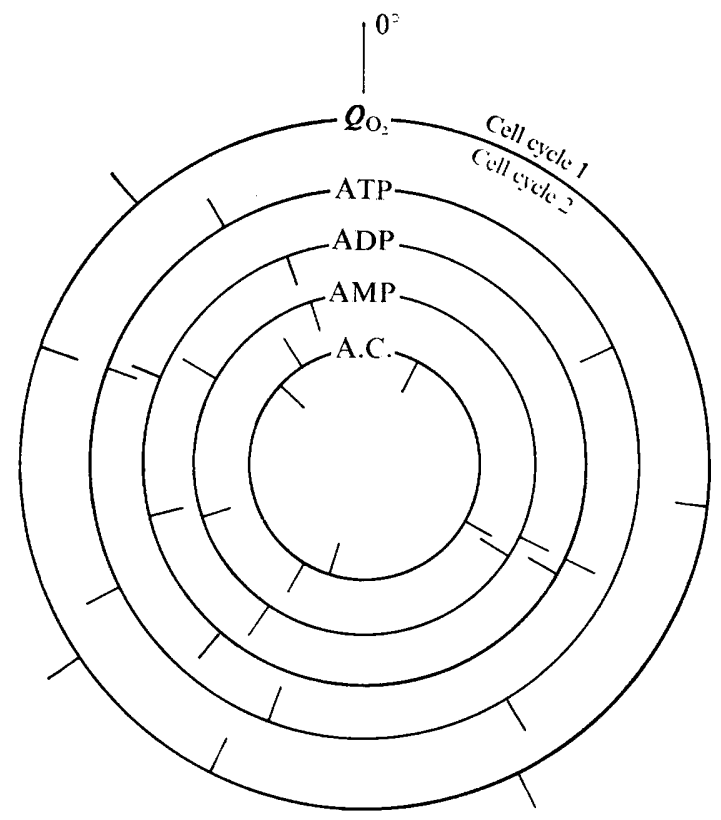

Fig. 6. Cell cycle map of the timings of maxima of oxygen uptake rates $\left(Q_{\mathrm{O}_{2}}\right)$, ATP, ADP and AMP pool sizes, and adenylate charge (A.C.) in T. pyriformis. The phase angles of these components are plotted with respect to the mid-points of cell division (at a phase angle of $0^{\circ}$ ). On each circle, the outer markers indicate maxima in the first cell cycle, and the inner markers, those in the second cell cycle. Results are expressed as the means of seven experiments for respiration rates and five experiments for adenine nucleotides.

showed oscillations. In the second cell cycle, the maxima occurred about $15 \mathrm{~min}$ in advance of the maxima of oxygen uptake rate (Fig. $5 b$ ). The amplitude of the calorimetrically measured oscillations was only 5 to $10 \%$ of the total rate of heat production at any time.

\section{DISCUSSION}

The oscillatory pattern of respiratory activity observed in synchronous cultures of $T$. pyriformis prepared by continuous-flow size selection contrasts with the exponential increase followed by terminal plateau found in heat-shock synchronized organisms (Zeuthen \& Scherbaum, 1954), or the exponential increase reported from measurements on single cells (Løvlie, 1963). Respiratory oscillations have previously been described during synchronous growth of the fission yeast Schizosaccharomyces pombe (Poole et al., 1973) and in the trypanosome Crithidia fasciculata (Edwards et al., 1975). The low values for adenylate charge calculated for both asynchronous and synchronous cultures growing at maximum rates under optimum conditions is remarkable and contrasts with the situation found in many other eukaryotes (Knowles, 1977; Chapman \& Atkinson, 1977). However, Edwards \& Lloyd (1977) have shown that Acanthamoeba castellanii can also grow at maximum rates when the adenylate energy charge value is extremely low (less than $0 \cdot 1$ ). It is evident that the value of adenylate charge in highly compartmented eukaryotes represents a spatially averaged value, and further work is necessary to determine the distribution of adenine nucleotides between the various intracellular compartments, particularly their partitioning between intra- and extra-mitochondrial spaces.

The decreased ATP pool previously noted at division of heat-shock synchronized $T$. pyriformis (Plesner, 1964; Stocco \& Zimmerman, 1975) was not observed in synchronous 
cultures prepared by continuous-flow size selection. However it has been shown that the nucleotide pool is affected by the heat treatment itself (De Balros et al., 1973); therefore comparisons between the two synchrony systems are not valid.

A summary of the mean values of timings of maxima of respiration and adenine nucleotides (Fig. 6) reveals that respiration rates oscillated in phase with ATP. This was also observed with $C$. fasciculata (Edwards et al., 1975). ADP and AMP pools oscillated in phase with each other, while no clear phase relationship was found between adenylate charge and any other parameter. These results indicate that the observed changes in adenylate pools cannot be explained in terms of mitochondrial respiratory control [i.e. the limitation of respiration rates by ADP (Chance \& Williams, 1956; Lloyd, 1974)] and further investigation is required to determine the control mechanisms involved in the generation of these slow, cell cycle-dependent oscillations.

We are indebted to Mrs L. John for technical assistance, to LKB Instruments Ltd for the loan of the microcalorimeter, and to Mr R. L. Taylor for his help. C.A.P. held a University of Wales postgraduate studentship and M.S. a Science Research Council studentship.

\section{REFERENCES}

Atkinson, D. E. \& Walton, G. M. (1967). Adenosine triphosphate conservation in metabolic regulation. Journal of Biological Chemistry 193, 265-275.

BALl, W. J. \& AtKinson, D. E. (1975). Adenylate energy charge in Saccharomyces cerevisiae during starvation. Journal of Bacteriology 121, 975-982.

Blumenthal, L. K. \& Zahler, S. A. (1962). Index for measurement of synchronization of cell populations. Science 135, 724.

Chance, B. \& Williams, G. R. (1956). The respiratory chain and oxidative phosphorylation. Advances in Enzymology 17, 65-134.

Chapman, A. G. \& Atkinson, D. E. (1977). Adenine nucleotide concentrations and turnover rates. Their correlation with biological activity in bacteria and yeast. Advances in Microbial Physiology 15, 253-306.

Chapman, A. G., Fall, L. \& Atkinson, D. E. (1971). Adenylate energy charge in Escherichia coli during growth and starvation. Journal of Bacteriology 108, 1072-1086.

de Balros, A. V., De Castro, J. F. \& De Castro, F. J. (1973). The effect of temperature on nucleotide pool formation in Tetrahymena pyriformis. Journal of Cellular Physiology 81, 149-152.

Dietzler, D. N., Lais, C. J., Magnani, J. L. \& LECKIE, M. P. (1974). Maintenance of the energy charge in the presence of large decreases in the total adenylate pool of Escherichia coli and concurrent changes in glucose-6-P, fructose- $\mathrm{P}_{2}$ and glycogen synthesis. Biochemical and Biophysical Research Communications 60, 875-881.

EdWards, S. W. \& Lloyd, D. (1977). Changes in oxygen uptake rates, enzyme activities, cytochrome amounts and adenine nucleotide pool levels during growth of Acanthamoeba castellanii in batch culture. Journal of General Microbiology 102, 135-144.

Edwards, C., Statham, M. \& Lloyd, D. (1975). The preparation of large-scale synchronous cultures of the trypanosomatid, Crithidia fasciculata, by cell-size selection: changes in respiration and adenylate charge through the cell-cycle. Journal of General Microbiology 88, 141-152.

KNowles, C. J. (1977). Microbial metabolic regulation by adenine nucleotide pools. Symposia of the Society for General Microbiology 27, 241283.

LLOYD, D. (1974). Environmental modification of mitochondrial composition and activities. In The Mitochondria of Microorganisms, pp. 159-250. London: Academic Press.

Lloyd, D., Brightwell, R., Venables, S. E., RoACH, G. I. \& Turner, G. (1971). Subcellular fractionation of Tetrahymena pyriformis ST by zonal centrifugation: changes in activities and distribution of enzymes during the growth cycle and on starvation. Journal of General Microbiology 65, 209-223.

Lloyd, D., John, L., Edwards, C. \& Chagla, A. H. (1975). Synchronous cultures of microorganisms: large-scale preparation by continuousflow size selection. Journal of General Microbiology 88, 153-158.

Lloyd, D., Morgan, N. A., John, L. \& Venables, S. E. (1978). Starvation of Prototheca zopfii. Journal of General Microbiology 105, 1-10.

LøvLIE, A. (1963). Growth in mass and respiration rate during the cell cycle of Tetrahymena pyriformis. Comptes Rendus des travaux du Laboratoire Carlsberg 33, 377-413.

Niven, D. F., Collins, P. A. \& Knowles, C. J. (1977). Adenylate energy charge during batch culture of Beneckea natriegens. Journal of General Microbiology 98, 95-108.

Phillips, C. A. \& Lloyd, D. (1978). Continuousflow size selection of Tetrahymena pyriformis strain ST: changes in volume, DNA, RNA and protein during synchronous growth. Journal of General Microbiology 105, 95-103.

Plesner, P. (1964). Nucleotide metabolism during synchronized cell division in Tetrahymena pyriformis. Comptes Rendus des travaux du Laboratoire Carlsberg 34, 1-76. 
Poole, R. K., Lloyd, D. \& Kemp, R. B. (1973). Respiratory oscillations and heat evolution in synchronously dividing cultures of the fission yeast Schizosaccharomyces pombe $972 \mathrm{~h}^{-}$. Journal of General Microbiology 77, 209-220.

Scherbaum, O. H., Chou, S. C., Seraydarian, K. H. \& BYFIELD, J. E. (1962). The effect of temperature shifts on the intracellular level of nucleoside triphosphates in Tetrahymena pyriformis. Canadian Journal of Microbiology 8, 753760.

Stocco, D. M. \& Zimmerman, A. M. (1975). Adenine nucleotide metabolism in heat-synchronized Tetrahymena. Molecular and Cellular Biochemistry 7, 187-194.
Swedes, J. S., Sedo, R. J. \& Atkinson, D. E. (1975). Relation of growth and protein synthesis to the adenylate energy charge in an adenine-requiring mutant of Escherichia coli. Journal of Biological Chemistry 250, 6930-6938.

ZeUthen, E. \& Scherbaum, O. (1954). Synchronous divisions in mass cultures of the ciliate protozoan Tetrahymena pyriformis as induced by temperature changes. In Recent Developments in Cell Physiology, Proceedings of the 7th Symposium of the Colston Research Society, pp. 141-156. Edited by J. A. Kitching. London: Butterworths. 